Departamento de Ciência e Tecnologia, Secretaria de Ciência, Tecnologia e Insumos Estratégicos, Ministério da Saúde

Correspondência | Correspondence:

Decit - Departamento de Ciência e Tecnologia do Ministério da Saúde

Esplanada dos Ministérios

Bloco G sala 845

70058-900 Brasília, DF, Brasil

Texto de difusão técnico-científica do Ministério de Saúde.

\section{Pesquisa Saúde: facilitando a apropriação do conhecimento científico na gestão de saúde}

\section{Health research: making easier the appropriation of scientific knowledge in health management}

Produzido em 2007 pelo Departamento de Ciência e Tecnologia (Decit) da Secretaria de Ciência, Tecnologia e Insumos Estratégicos do Ministério da Saúde, o Pesquisa Saúde é um sistema disponível em ambiente web, interativo e de acesso livre.

O objetivo do Pesquisa Saúde é aproximar dos gestores o conhecimento científico produzido, auxiliando a tomada de decisão e abreviando obstáculos como a burocracia, a morosidade e a dificuldade de encontrar resultados e soluções precisas para os problemas de saúde.

O sistema é composto de uma base de dados que reúne um universo de 3 mil pesquisas em temas prioritários de saúde no País e pode ser acessado por estudantes, profissionais da área, pesquisadores e sociedade civil do Brasil e do mundo no portal do Ministério da Saúde.

A maior parte dos assuntos é distribuída nas 24 subagendas da Agenda Nacional de Prioridade de Pesquisa em Saúde (ANPPS), documento norteador do financiamento de estudos do Decit. Além do elenco de temas da ANPPS, outras áreas de relevância em saúde, como células-tronco, neoplasias, avaliação de tecnologias em saúde, doenças negligenciadas, estão na base de dados. O documento orienta as ações de fomento à pesquisa de acordo com as necessidades de saúde da população brasileira.

A partir de informações comuns à prática de fomento, como o valor investido em pesquisa, o ano do edital, o estado contemplado, a natureza da pesquisa, as modalidades de fomento, entre outras, o sistema permite gerar indicadores e gráficos, bem como exportar dados em planilha eletrônica. Tal ferramenta pode auxiliar pesquisadores a encontrar estudos semelhantes ao que desenvolvem e ser fonte de pesquisa para especialistas em saúde.

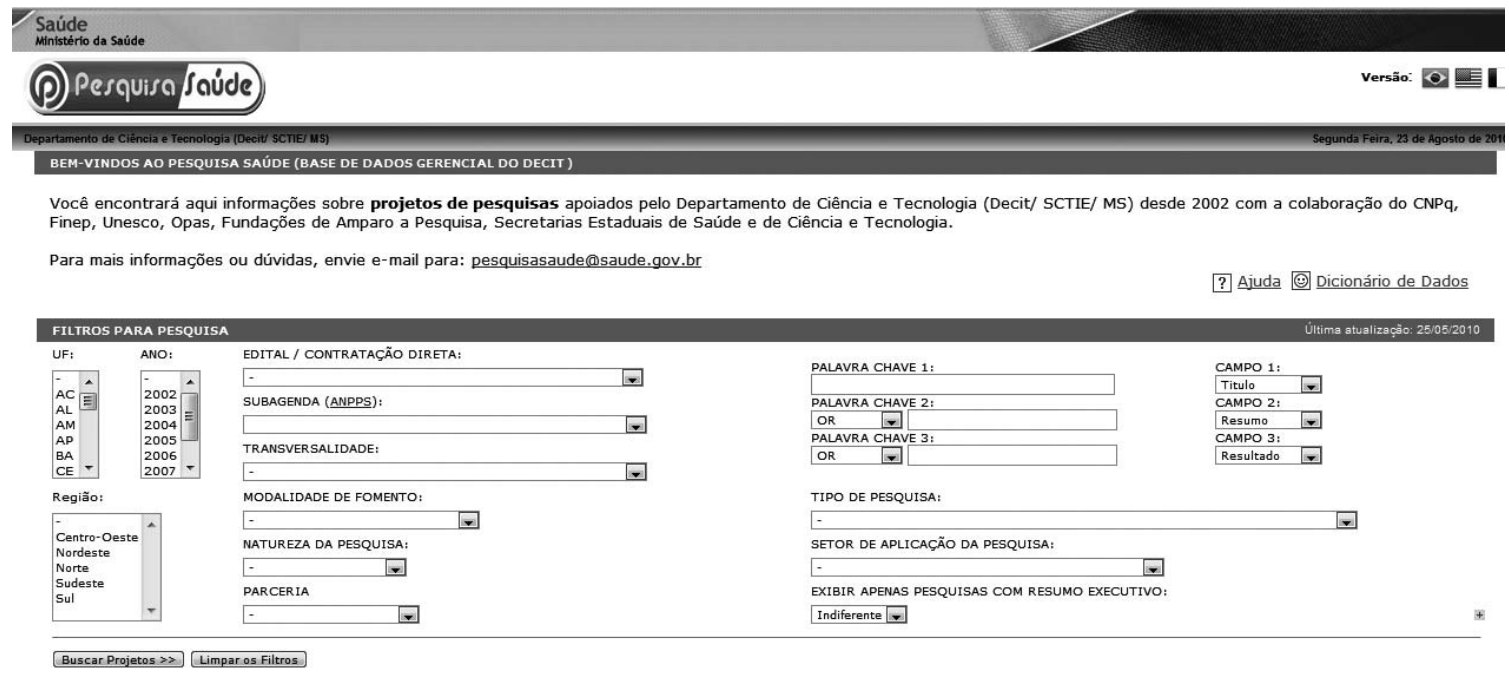

Figura 1. Página inicial do Pesquisa Saúde, com os campos de informação. 


\begin{tabular}{|c|c|c|c|c|}
\hline $\begin{array}{l}\text { Quadro - Número de Projetos e Recursos por Su } \\
\text { Subagenda }\end{array}$ & $\begin{array}{l}\text { enda } \\
\text { No de } \\
\text { Projetos }\end{array}$ & $\%$ & Total Recursos & $\%$ \\
\hline ALI MENTAÇÃO E NUTRIÇÃO & 226 & 7.4 & $\mathrm{R} \$ 11.495 .713,24$ & 2.2 \\
\hline ASSISTÊNCIA FARMACÊUTICA & 139 & 4.6 & $\mathrm{R} \$ 18.794 .957,29$ & 3.5 \\
\hline AVALIAÇÃO DE TECNOLOGIAS E ECONOMIA DA SAÚDE & 221 & 7.3 & $\mathrm{R} \$ 23.399 .038,96$ & 4.4 \\
\hline BIOÉTICA E ÉTICA EM PESQUISA & 154 & 5.1 & $\mathrm{R} \$ 3.590 .302,26$ & 0.7 \\
\hline COMPLEXO PRODUTIVO DA SAÚDE & 112 & 3.7 & $\mathrm{R} \$ 146.439 .330,13$ & 27.4 \\
\hline COMUNICAÇÃO E INFORMAÇÃO EM SAÚDE & 58 & 1.9 & $\mathrm{R} \$ 8.394 .068,73$ & 1.6 \\
\hline DEMOGRAFIA E SAÚDE & 2 & 0.1 & $\mathrm{R} \$ 7.834 .842,07$ & 1.5 \\
\hline DOENÇAS CRÔNICAS (NÃO-TRANSMISSIIVEIS) & 294 & 9.6 & $\mathrm{R} \$ 53.617 .770,60$ & 10 \\
\hline DOENÇAS TRANSMISSIIVEIS & 555 & 17.1 & $\mathrm{R} \$ 89.748 .703,35$ & 12.8 \\
\hline EPIDEMIOLOGIA & 25 & 0.8 & $\mathrm{R} \$ 5.702 .912,22$ & 1.1 \\
\hline GESTÃO DO TRABALHO E EDUCAÇÃO EM SAÚDE & 88 & 2.9 & $\mathrm{R} \$ 8.791 .791,10$ & 1.6 \\
\hline PESQUISA CLÍNICA & 190 & 6.3 & $\mathrm{R} \$ 94.507 .300,65$ & 17.8 \\
\hline PROMOÇÃO DA SAÚDE & 14 & 0.5 & $\mathrm{R} \$ 746.682,31$ & 0.1 \\
\hline SAÚDE BUCAL & 103 & 3.4 & $\mathrm{R} \$ 4.320 .361,48$ & 0.8 \\
\hline SAÚDE DA CRIANÇA E DO ADOLESCENTE & 108 & 3.6 & $\mathrm{R} \$ 4.820 .626,77$ & 0.9 \\
\hline SAÚDE DA MULHER & 147 & 4.8 & $\mathrm{R} \$ 14.769 .828,08$ & 2.8 \\
\hline SAÚDE DA POPULAÇÃO NEGRA & 30 & 1 & $\mathrm{R} \$ 2.911 .856,09$ & 0.5 \\
\hline SAÚDE DO IDOSO & 41 & 1.3 & $\mathrm{R} \$ 7.775 .124,69$ & 1.5 \\
\hline SAÚDE DOS PORTADORES DE NECESSIDADES ESPECIAIS & 27 & 0.9 & $\mathrm{R} \$ 2.016 .558,72$ & 0.4 \\
\hline SAÚDE DOS POVOS INDÍGENAS & 59 & 2 & $\mathrm{R} \$ 3.191 .469,74$ & 0.6 \\
\hline SAÚDE MENTAL & 133 & 4.4 & $\mathrm{R} \$ 13.191 .610,16$ & 2.5 \\
\hline SAÚDE, AMBIENTE, TRABALHO E BIOSSEGURANÇA & 72 & 2.4 & $R \$ 9.873 .916,32$ & 1.8 \\
\hline SISTEMAS E POLÍTICAS DE SAÚDE & 185 & 6.1 & $\mathrm{R} \$ 12.901 .546,76$ & 2.4 \\
\hline VIOLÊNCIA, ACIDENTES E TRAUMA & 85 & 2.8 & $\mathrm{R} \$ 5.742 .275,67$ & 1.1 \\
\hline Total: 24 Subagenda(s) & 3073 & 100 & $\mathrm{R} \$ 554.823 .015,99$ & 100 \\
\hline
\end{tabular}

Fonte: Brasil, Ministério da Saúde, Departamento de Ciência e Tecnologia - Decit. Base de Dados Gerencial. Capturado em 23/08/2010

Figura 2. Exemplo de indicador, com número de projetos e recursos financeiros por subagendas da Agenda Nacional de Prioridade de Pesquisa em Saúde.

\section{HISTÓRICO}

O Decit, ao longo dos seus dez anos de atividade, consolidou seu papel no cenário da ciência e tecnologia em saúde em contexto nacional, sobretudo, pelas parcerias institucionais estabelecidas com o Ministério de
Ciência e Tecnologia, por meio do Conselho Nacional de Desenvolvimento Científico e Tecnológico (CNPq), da Financiadora de Estudos e Projetos (Finep) e das Fundações de Amparo à Pesquisa, além das Secretarias Estaduais e Municipais de Saúde. Também são parceiras do Departamento a Organização das Nações Unidas

\begin{tabular}{lcl}
$\begin{array}{l}\text { Quadro - Número de Projetos e Recursos por Subagenda (10 Maiores em No de } \\
\text { Proietos) } \\
\text { Subagenda }\end{array}$ & No de Projetos & Total Recursos \\
\hline DOENÇAS TRANSMISSÍVEIS & 555 & $\mathrm{R} \$ 89.748 .703,35$ \\
DOENÇAS CRÔNICAS (NÃO-TRANSMISSÍVEIS) & 294 & $\mathrm{R} \$ 53.617 .770,60$ \\
ALIMENTAÇÃO E NUTRIÇÃO & 226 & $\mathrm{R} \$ 11.495 .713,24$ \\
AVALIAÇÃO DE TECNOLOGIAS E ECONOMIA DA SAÚDE & 221 & $\mathrm{R} \$ 23.399 .038,96$ \\
PESQUISA CLÍ IICA & 190 & $\mathrm{R} \$ 94.507 .300,65$ \\
SISTEMAS E POLÍTICAS DE SAÚDE & 185 & $\mathrm{R} \$ 12.901 .546,76$ \\
BIOÉTICA E ÉTICA EM PESQUISA & 154 & $\mathrm{R} \$ 3.590 .302,26$ \\
SAÚDE DA MULHER & 147 & $\mathrm{R} \$ 14.769 .828,08$ \\
ASSISTÊNCIA FARMACÊUTICA & 139 & $\mathrm{R} \$ 18.794 .957,29$ \\
SAÚDE MENTAL & 133 & $\mathrm{R} \$ 13.191 .610,16$ \\
Total & 2244 & $\mathrm{R} \$ 336.016 .771,35$ \\
\hline
\end{tabular}

Fonte: Brasil, Ministério da Saúde, Departamento de Ciência e Tecnologia - Decit. Base de Dados Gerencial. Capturado em 23/08/2010 


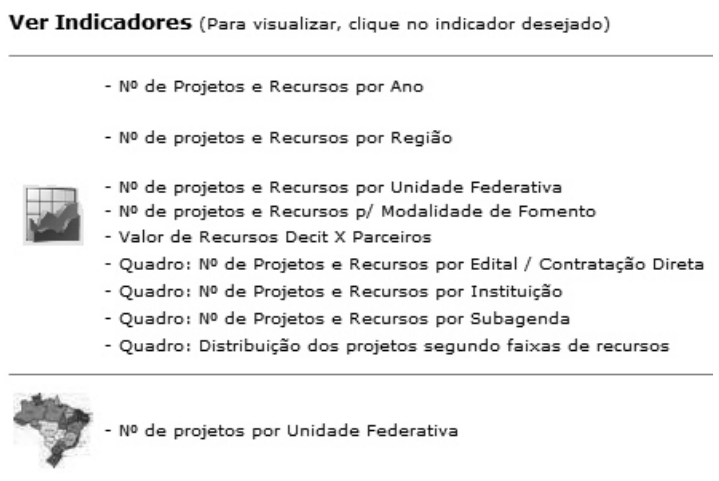

Figura 4. Relação de indicadores do sistema.

para a Educação, a Ciência e a Cultura (Unesco) e a Organização Pan-Americana de Saúde (Opas). Esse esforço conjunto assegura que os recursos direcionados para as ações de fomento à pesquisa respondam às demandas de saúde peculiares de cada estado, contribuindo para a superação das desigualdades regionais em saúde.

Todos os estados do Brasil já foram contemplados com recursos em pesquisa pelo Decit, inclusive os da região Nordeste, que geralmente costumam ter mais dificuldades de acesso aos investimentos e, conseqüentemente, em produzir estudos científicos. Os Estados da Bahia (5 lugar) e de Pernambuco ( $6^{\circ}$ ) estão entre os dez que mais receberam recursos em pesquisa de 2002 a 2008, com R \$ 35,4 milhões e R \$ 24,2 milhões, respectivamente. Em primeiro lugar, está São Paulo (R\$ 142,2 milhões), seguido do Rio de Janeiro (R\$ 129,3 milhões), Rio Grande do Sul (R\$ 57,2 milhões) e Minas Gerais (R\$
44,7 milhões). Dessa forma, o Sudeste é a região que mais concentra investimentos em pesquisas. O Estado do Acre foi o que menos recebeu recursos, num valor de $\mathrm{R} \$ 550,7$ mil para 13 projetos.

\section{ESTRATÉGIAS}

A Política Nacional de Ciência, Tecnologia e Insumos Estratégicos, mais que uma conquista no setor da saúde, orienta e respalda as muitas ações do Decit, como a Difusão dos Avanços Científicos e Tecnológicos. Nessa perspectiva, o Pesquisa Saúde facilitou a construção de outro sistema com a mesma plataforma gerencial, o Sistema de Informação em Ciência, Tecnologia e Inovação em Saúde. Nesse sistema, podem ser consultadas todas as fases do processo de fomento, desde a construção de editais, as inscrições dos projetos, a participação das comissões julgadoras até os resultados da pesquisa. Os prêmios de Incentivo em Ciência e Tecnologia para o Sistema Único de Saúde e o de IncentivoàPromoçãodo Uso Racional de Medicamentos também têm no sistema suas etapas de inscrição de trabalhos, avaliação de pareceristas ad hoc, entre outras.

Desde seu funcionamento, em 2008, o Pesquisa Saúde registrou acessos ao sistema como forma de aferir seu desempenho. Saber quem o consulta, de onde é realizado o estudo e quanto tempo dura a pesquisa são dados que certificam a funcionalidade da ferramenta. Dessa forma, uma pesquisa realizada pelos técnicos do sistema mostrou que, entre os dias 22 de dezembro de 2008 e 23 de março de 2009, o Pesquisa Saúde foi acessado 2.847 vezes por 2.359 visitantes. A média de tempo gasto em

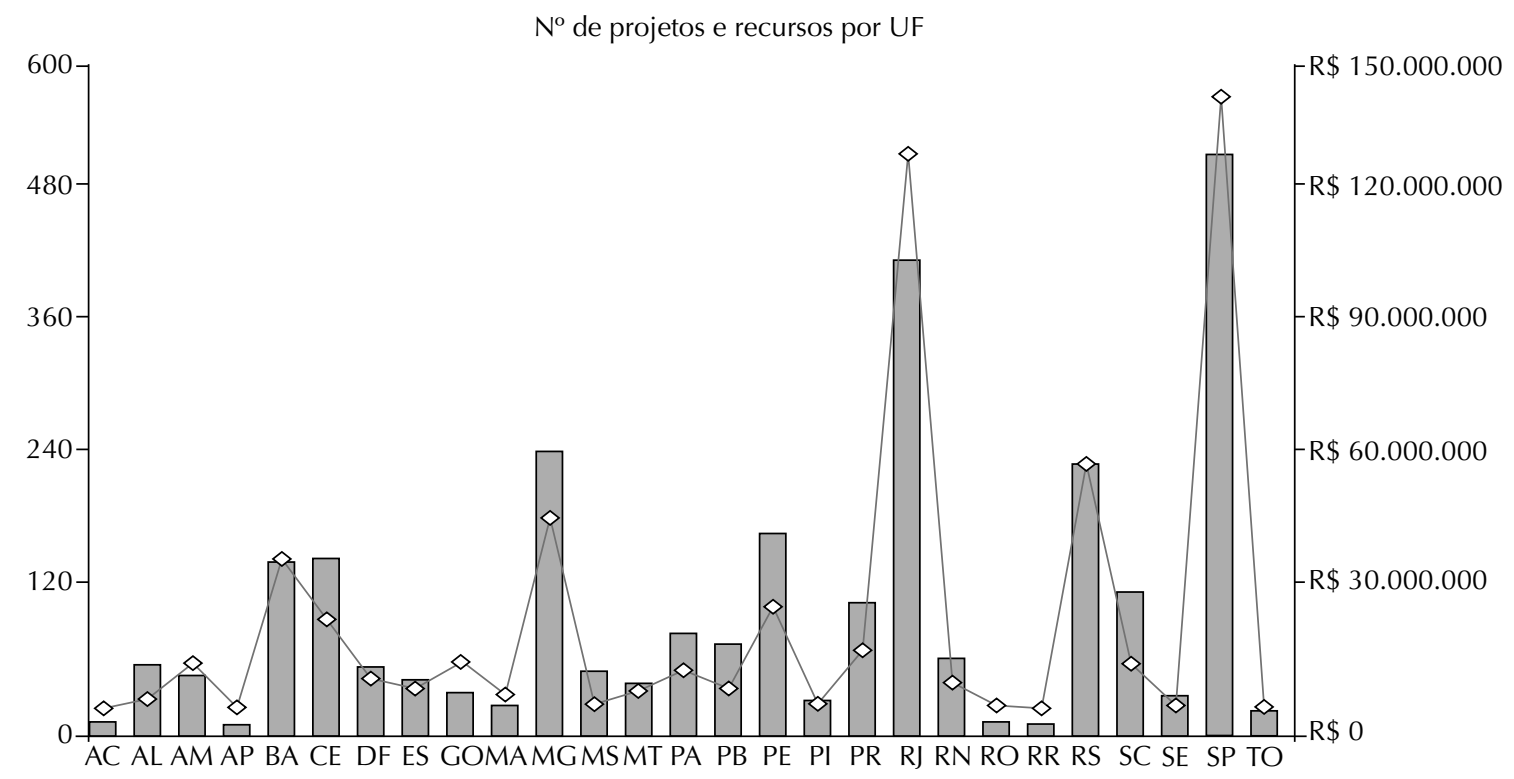

$\square \mathrm{N}^{\mathrm{o}}$ de Projetos — Recurso Total (Em Milhões)

Figura 5. Recursos financeiros destinados pelo Decit e parceiros institucionais por região geográfica, 2003 a 2008. 
cada acesso é de três minutos e 34 segundos, o que pode indicar que os usuários fazem mais consultas pontuais e específicas na base de dados.

As visitas são originárias de 20 países. Além do Brasil, a Suíça, os Estados Unidos, a Espanha, a Inglaterra e Portugal são os países que mais acessaram o sistema. No Brasil, São Paulo foi a cidade que mais gerou acessos (427), seguida do Rio de Janeiro (314), Brasília (238) e Belo Horizonte (205).

Uma pesquisa conduzida pelo George Institute for Internacional Health, a G-Finder, que investigava o rastreamento global em pesquisa e desenvolvimento em doenças negligenciadas, recorreu ao Decit, solicitando todos os dados da temática pelo Pequisa Saúde.
Posteriormente, o Council on Health Research for Development (Cohred) iniciou, junto ao Decit, o desenvolvimento de um sistema informatizado semelhante ao Pesquisa Saúde, utilizando-o como referência para sua construção. O Cohred é uma organização internacional, não-governamental, cujo trabalho consiste no apoio a países em desenvolvimento para a construção de sistemas necessários à realização de pesquisas em saúde.

A ferramenta do Pesquisa Saúde é única e de importância singular no cenário de ciência e tecnologia do Brasil e do mundo. Atualmente, o acesso à base de dados é universal, possibilitando transparência às ações desenvolvidas pelo Decit e parceiros, além dos pesquisadores que buscam informações para produzir avanços na saúde tanto do País quanto mundialmente. 